\title{
SIR MODEL OF TIME DEPENDENT DRUG AND VACCINE DISTRIBUTION ON COVID-19*
}

\author{
MEGAN JOHNSTON , LAIRD STEWART , JESSE SUN , AND DAVID ZHANG †
}

Abstract. Since the end of 2019, COVID-19 has threatened human life around the globe. As the death toll continues to rise, development of vaccines and antiviral treatments have progressed at unprecedented speeds. This paper uses an SIR-type model, extended to include asymptomatic carrier and deceased populations as a basis for expansion to the effects of a time-dependent drug or vaccine. In our model, a drug is administered to symptomatically infected individuals, decreasing recovery time and death rate. Alternatively, a vaccine is administered to susceptible individuals and, if effective, will move them into the recovered population. We observe final mortality outcomes of these countermeasures by running simulations across different release times with differing effectivenesses.

As expected, the earlier the drug or vaccine is released into the population, the smaller the death toll. We find that for earlier release dates, difference in the quality of either treatment has a large effect on total deaths. However as their release is delayed, these differences become smaller. Finally, we find that a vaccine is much more effective than a drug when released early in an epidemic. However, when released after the peak of infections, a drug is marginally more effective in total lives saved.

Key words. COVID-19, Coronavirus, SARS-CoV-2, Drug, Vaccine, SIR, Simulation

1. Introduction. The world faces an unknown future due to SARS-CoV-2. In the U.S., discussion surrounds re-opening, social distancing measures, and a second wave. Researchers have made strides in the development of drugs and vaccines at unprecedented speeds, but the virus continues to spread with U.S. infections and deaths increasing every day [4]. Figure 1 shows the total number of deaths and cases in the US since the start of 2020 .
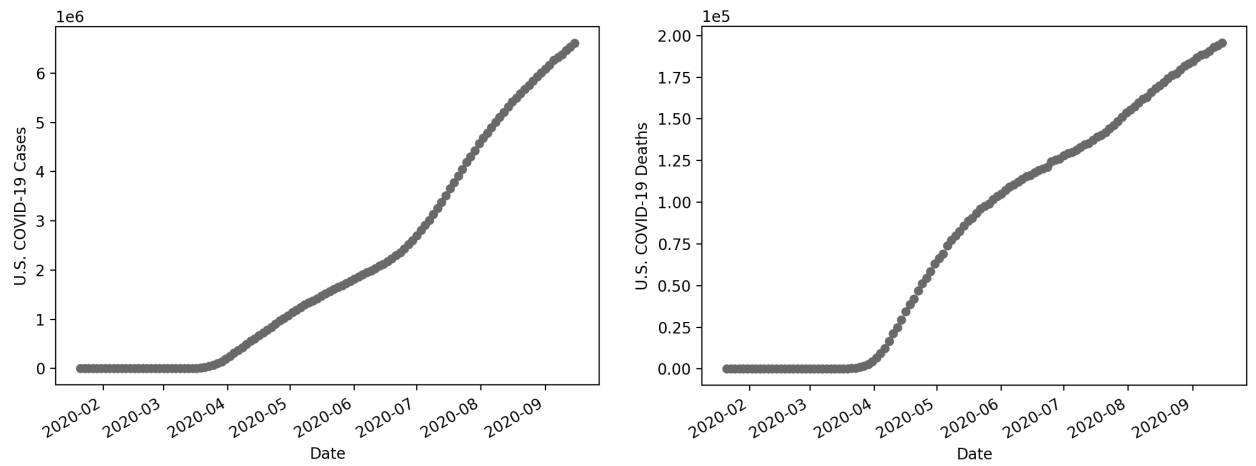

FIG. 1. Total U.S. COVID-19 Cases (Left), and Deaths (Right)[19]

One of the United States' major responses to the pandemic has been Operation Warp Speed (OWS). The partnership between the CDC, FDA, NIH, DoD and others

* Submitted to the editors September 26, 2020.

Funding: This paper is the follow-up to a submission for a contest held by Emory University's Department of Mathematics in April 2020. The authors were awarded first place prize in the contest.

$\dagger$ Department of Mathematics, Emory University. Undergraduate Majors in Applied Math. Megan Johnston (Sr.) megan.irene.johnston@emory.edu, Laird Stewart (Jr.) laird.stewart@emory.edu, David Zhang (Jr.) david.zhang@emory.edu, and Jesse Sun (So.) jesse.sun@emory.edu.

Faculty Sponsor: Alessandro Veneziani, Emory Department of Mathematics and Computer Science. avenez2@emory.edu 
has committed to providing 300 million doses of a vaccine by the end of January 2021. OWS has provided more than $\$ 2$ billion in funding for vaccines to Johnson \& Johnson, Moderna, and AstraZeneca/Oxford [2]. As of September 26th, each of these trials are in phase 3 [7].

While OWS has not funded antiviral development, companies around the world are also racing to develop antivirals. Antivirals from Gilead Sciences (Remdesivir), AstraZeneca, and Merck \& Co. are currently authorized, in phase 1, and in phase 2, respectively [7]. These treatments' mechanisms and deliveries vary but have all shown promise.

Because vaccines and drugs function in different ways and are administered to different populations, their effects on the pandemic will not be the same. Outcomes will also depend on the pharmacological effectivenesses of the countermeasures as well as the speed and date of their introduction.

In this work, we consider a basic mathematical model extended to provide insight into these outcomes. The model takes into account the time-dependent distribution of a drug or vaccine and respective patient outcomes (e.g. the rate of recovery or death). It can be expanded to fit any population or disease variable, but has been fit to those of the U.S. for the sake of this work.

Our model is based on a standard set of SIR equations for susceptible, infected and recovered populations. An SIR model is a system of ordinary differential equations which describes how an outbreak spreads through a population [18]. It is comprised of the following three equations:

$$
\begin{aligned}
\frac{d S}{d t} & =-\beta I\left(\frac{S}{N}\right) \\
\frac{d I}{d t} & =\beta I\left(\frac{S}{N}\right)-\gamma I \\
\frac{d R}{d t} & =\gamma I
\end{aligned}
$$

$S, I$, and $R$ are the number of susceptible, infected, and recovered people at time $t$, respectively. The problem is completed by appropriate initial conditions $S_{0}, I_{0}$, and $R_{0}$. At any time the size of the population, $N$, is defined as the following:

$$
N=S+I+R
$$

In this system, $\beta$ is the expected number of people an infected person infects per day. It is closely related to the so-called $R_{0}$ or ' $\mathrm{R}$ naught' which is the total number of people that one infected person will infect over the duration of their illness. Therefore, $\beta$ is $R_{0} / L$ where $L$ is the duration of the illness. The rate at which the infected group recovers is $\gamma$. Gamma can also be expressed as $1 / L$ because, for example, if it takes 5 days to recover then $\gamma=0.2$ as one in five infected individuals will recover each day [11].

While this basic model is indicative of important dynamics, it is too crude for our problems because it does not consider asymptomatic or deceased individuals who are important in the context of COVID-19. For this reason, we introduce an expanded SIR model as a Basis Model for further analysis.

The paper is organized as follows: Our Basis Model is in section 2, our model including a drug is in section 3, our model including a vaccine is in section 4 , our final results are in section 5 , and a discussion follows in section 6 . 


\section{COVID-19 Basis Model.}

2.1. Equations. Using the SIR model as a starting point, its equations are expanded to include asymptomatic carrier $(A)$ and deceased $(D)$ populations [11]. These will be critical when expanding the model for a drug and vaccine release. The total population therefore becomes the following:

$$
N=S+A+I+R+D
$$

The flow chart representing the different compartments of our model and their interactions is shown in Figure 2:

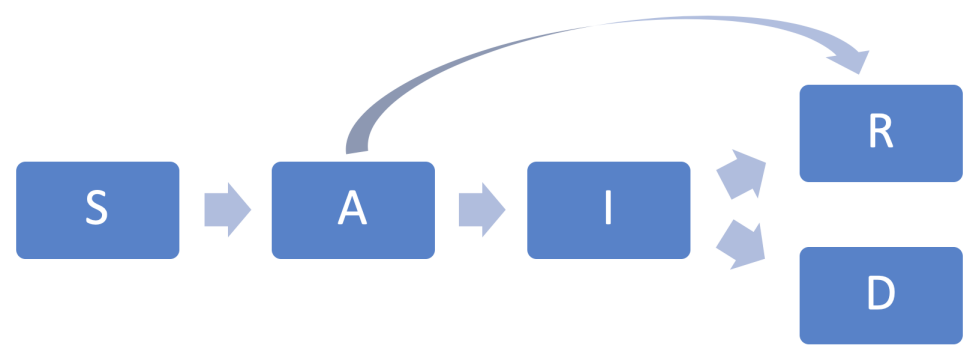

FIG. 2. Compartmental Flow Chart for Base SAIRD Model

With the inclusion of an asymptomatic carrier population, there are two groups with different risks of infecting others. Therefore, $\beta$ is split into $\beta_{i}$ and $\beta_{a}$ for the symptomatically infected and asymptomatic carrier populations, respectively. Therefore change in the susceptible population becomes the following:

$$
\frac{d S}{d t}=-\left[\beta_{i} I\left(\frac{S}{N}\right)+\beta_{a} A\left(\frac{S}{N}\right)\right]
$$

Susceptible individuals will become asymptomatic carriers $(A)$ before becoming symptomatically infected $(I)$ so the positive rate of change for the asymptomatic population, $\frac{d A}{d t}$, will be the opposite of the rate of change of the susceptible population. Asymptomatic individuals will either develop symptoms or recover without showing symptoms. Let $\mu$ be the proportion of asymptomatic individuals who become symptomatically infected. Therefore, the total change in the asymptomatic population can be defined as the following:

$$
\frac{d A}{d t}=\left[\beta_{i} I\left(\frac{S}{N}\right)+\beta_{a} A\left(\frac{S}{N}\right)\right]-\mu A n_{i}-(1-\mu) A n_{r}
$$

where $n_{i}$ is the rate at which people transition from asymptomatic carriers to symptomatically infected in one day. In other words, 1 /(days to transition to symptomatically infected from asymptomatic). Further, $n_{r}$ is the rate at which people transition from asymptomatic to recovered. In other words $1 /$ (days to transition to recovered from asymptomatic). Notice that if $n_{i}=n_{r}=n$, the negative rate of change would simply become $-A n$.

Increases in the symptomatically infected population $(I)$ will result from asymptomatic individuals who begin to show symptoms. Once symptomatic, individuals 
will either join the recovered or deceased populations. Let $\alpha$ represent the proportion of individuals who die from the virus. Moreover, let $\rho$ be the rate at which people die, or $1 /$ (days it takes to die if symptomatically infected). Also, let $\gamma$ be the rate at which people recover, or 1 ( (days it takes to recover from symptomatically infected). The change in symptomatically infected individuals, $I$, is therefore the following [11]:

$$
\frac{d I}{d t}=\mu A n_{i}-\alpha \rho I-(1-\alpha) \gamma I
$$

The change in the recovered population can be described using the components of the asymptomatic carrier and symptomatically infected populations who recover:

$$
\frac{d R}{d t}=(1-\mu) A n_{r}+(1-\alpha) \gamma I
$$

Similarly, the change in the deceased population can be described using the components of the symptomatically infected population who die:

$$
\frac{d D}{d t}=\alpha \rho I
$$

In summary, the model can be written in matrix form where $\boldsymbol{u}=[S, A, I, R, D]^{T}$ and summarized as the vector $\frac{d(u)}{d t}$, where each entry corresponds to each of the five equations:

$$
\begin{gathered}
\frac{\boldsymbol{d}(\boldsymbol{u})}{\boldsymbol{d} \boldsymbol{t}}=\boldsymbol{B}(\boldsymbol{u}) \boldsymbol{u} \\
\boldsymbol{B}(\boldsymbol{u})=\left[\begin{array}{ccccc}
0 & -\frac{\beta_{a} S}{N} & -\frac{\beta_{i} S}{N} & 0 & 0 \\
0 & \frac{\beta_{a} S}{N}-\mu n_{i}-(1-\mu) n_{r} & \frac{\beta_{i} S}{N} & 0 & 0 \\
0 & \mu n_{i} & -\alpha \rho-(1-\alpha) \gamma & 0 & 0 \\
0 & (1-\mu) n_{r} & (1-\alpha) \gamma & 0 & 0 \\
0 & 0 & \alpha \rho & 0 & 0
\end{array}\right]
\end{gathered}
$$

The matrix format allows for analysis of the model in its equilibrium state to understand the potential of achieving a state where there is no change in the populations from the vaccine or drug. The full system of equations is summarized in Appendix A.

2.2. Tuning Parameters. Values from current reports on SARS-CoV-2 provide potential values for our parameters. However, due to the novelty of SARS-CoV-2, it is important to note that these values are epidemiological estimates and continue to change.

The proportion of symptomatically infected patients that will show symptoms, $\mu$, varies widely among sources. In this paper it is taken as 0.85 , a rough estimate given a few studies $[8,9]$. In practice, changes in the value of $\mu$ within the range given by Buitrago-Garcia's meta-analysis of studies ([8]: 0.45-0.97), affect the final case and death counts but do not affect the shape of the curves or overall system dynamics. Therefore our analysis is not affected by the exact value of $\mu$.

The length of time before symptoms present themselves is approximately 5.1 days [13], so $n_{i}$ can be estimated at $\frac{1}{5.1}=0.1961$. Likewise, the number of days to transition to recovered from asymptomatic is estimated to be 9 days, therefore $n_{r}$ is $\frac{1}{9}=0.1111$ [17]. Next, the proportion of individuals who die from the virus, $\alpha$, is estimated at 
$0.64 \%$ with the length of time it takes to die estimated at 17.8 days $[15,20]$. Therefore, $\rho$ is estimated to be $\frac{1}{17.8}=0.0562$. Finally, the length of time it takes to recover is estimated to be between $10-13$ days [3], so $\gamma$ becomes $\frac{1}{11.5}=0.0870$.

The number of people infected by asymptomatic and symptomatically infected people per day are $\beta_{a}$ and $\beta_{i}$ respectively. To calculate these parameters, we take the total number of people an asymptomatic or symptomatically infected individual infects $\left(R_{0}\right)$ and divide by the duration of their illness. However, data about different rates of infectivity between asymptomatic carriers and symptomatically infected people is not widely published or agreed upon. Because of this, we use a single $\beta$ for both the asymptomatic and symptomatically infected populations. It is calculated as $\frac{R_{0}}{\text { duration }}$.

The quantity $R_{0}$ is the total expected number of people that one individual will infect over the course of the disease. Note that this is the 'base rate' infectivity of the virus and does not account for social distancing, masks, or other health precautions. The estimated value of $R_{0}$ is widely disputed. However, the World Health Organization suggests that the value lies between 1.4 and 2.5 , so we will approximate it as 2 [6]. Next we divide $R_{0}$ by a constant duration of infection. However, depending on whether or not an individual is symptomatic, this duration will change. As a heuristic, we approximate the duration of the disease as $1 / n_{i}+1 / \gamma$ because the majority of people will show symptoms and the vast majority of those will not die. Therefore, $\beta=R_{0} /\left(1 / n_{i}+1 / \gamma\right)$. This gives us $2 /(5.1+11.5)=0.1205$.

Finally, for the initial conditions of the model, the remainder of this paper will use $I_{0}=1000$ and $S_{0}=N-1000$ with the remaining initial populations set to zero. The total population, $N$, is that of the U.S.: 3.28196e8. These initial conditions assume that everyone in the population is susceptible to the virus. Small variations in the number of initially infected, $I_{0}$, around zero do not change dynamics of the system or our results, but determine how quickly the peak of infections occur.

Our estimates for these parameters can be summarized in Appendix D.

2.3. Equilibrium. We investigate the equilibrium and stability of our system of equations for a better assessment of our model. At the equilibrium in our model, each of the five differential equations equals zero under the assumption that the total population does not change. Therefore, the equilibrium is some $\boldsymbol{u}^{*}$ where $\boldsymbol{B}\left(\boldsymbol{u}^{*}\right) \boldsymbol{u}^{*}=0$. In our model, $n_{i}, n_{r}, \rho, \gamma, \beta_{a}$, and $\beta_{i}$ are greater than zero. Further, $\mu$ and $\alpha$ are greater than zero and less than one. Given these constraints, $I^{*}$ must equal zero to satisfy the equilibrium for equation (2.5). With this conclusion, $A^{*}$ must also equal zero to satisfy the equilibrium for equation (2.3). By setting $I^{*}$ and $A^{*}$ equal to zero, all of the differential equations satisfy equilibrium. This is also visually apparent in matrix (2.7). Therefore, the number of symptomatically infected and asymptomatic individuals must equal zero for there to be no further change in the populations. $S^{*}$, $R^{*}$, and $D^{*}$ can be any constants such that $S^{*}+R^{*}+D^{*}=N$. In summary, the equilibrium point $\boldsymbol{u}^{*}$ can be written as $\left[S^{*}, 0,0, R^{*}, D^{*}\right]^{T}$ where $S^{*}, R^{*}$ and $D^{*}$ are constants.

Using the estimated values for the constants, the eigenvalues can be solved for using a Jacobian Matrix (Appendix E). Three eigenvalues equal zero, one eigenvalue is negative, and the final eigenvalue is positive when $\frac{S_{0}}{N}>0.52$. When this condition is satisfied, the equilibrium is not asymptotically stable but rather a saddle point. When $S_{0}$ represents less than $52 \%$ of the total population, the fifth eigenvalue is negative and the equilibrium is asymptotically stable. When this is true, the initial conditions are already stable. This demonstrates that, if the population was $52 \%$ immune when 
COVID-19 began its spread, herd immunity would have already been achieved and the pandemic would not have occurred.
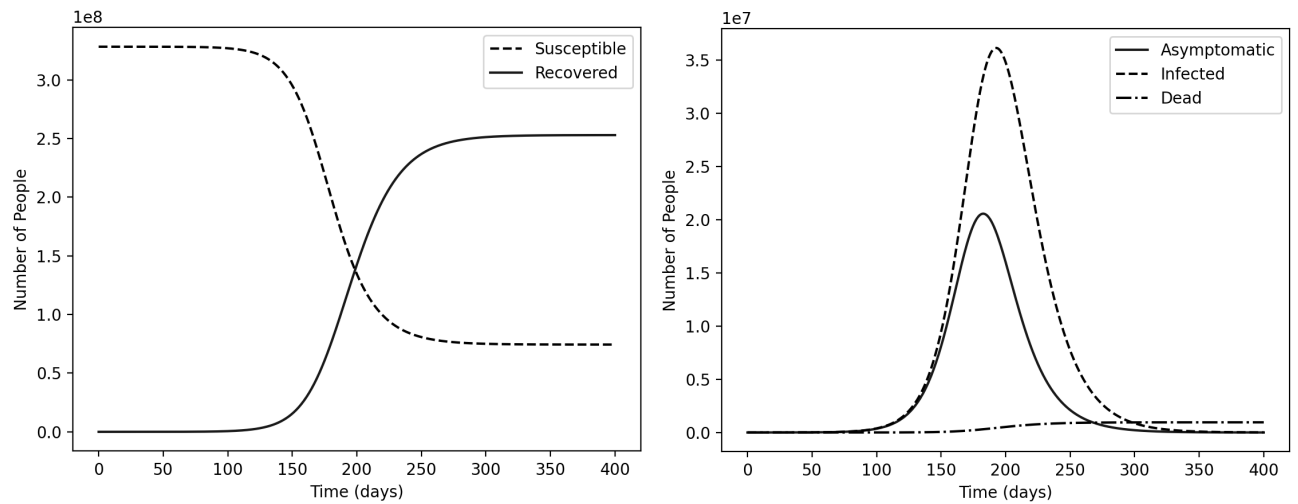

FIG. 3. Base SAIRD Trajectory

2.4. Results. As we would expect from an SIR-type model, the susceptible and recovered populations follow logistic curves as shown in Figure 3. The asymptomatic and symptomatically infectious curves peak and return to zero. The death curve is also logistic with an asymptotic value of 956,155 . The susceptible and recovered curves have inflection points close to the peak of the symptomatically infected curve. This is what we would expect, because after the number of infections peaks, the growth of the recovered population will slow. Conversely, the susceptible curve also flattens because less people are being infected. These inflection points and peaks will be critical to the results of a drug or vaccine.

\section{Inclusion of a Drug.}

3.1. Modeling the Drug. The inclusion of a drug only changes the model's parameters, not the overall structure of the equations. Once a drug is available, more of the symptomatically infected population will recover and less will die. This is shown in Figure 4. Recovery of the infected also becomes faster. Therefore $\frac{d S}{d t}$ and $\frac{d A}{d t}$ remain the same from the Basis Model.

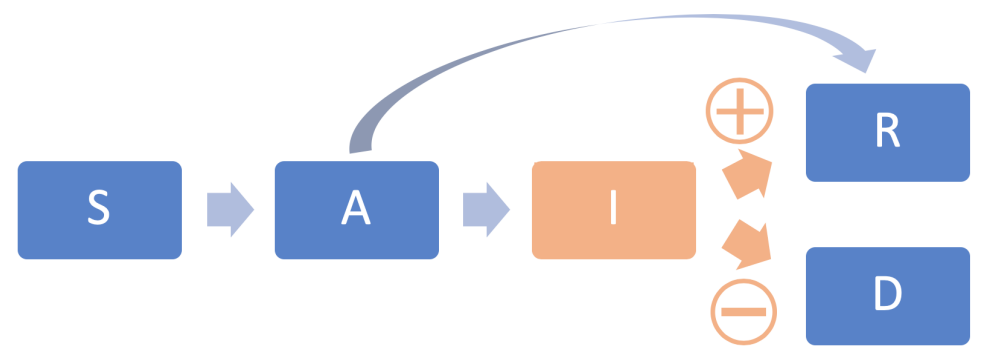

FIG. 4. Compartmental Flow Chart With Inclusion of Drug

The introduction of a drug will help some in the symptomatically infected population recover, but not all individuals will receive the drug. Therefore, the $\frac{d I}{d t}$ is 
partitioned by $j$, the percentage of individuals who receive the drug. The change in symptomatically infected individuals, $I$, becomes the following:

$$
\frac{d I}{d t}=\mu A n_{i}-\overbrace{(1-j) \alpha \rho I}^{\text {no drug, deceased }}-\underbrace{(1-j)(1-\alpha) \gamma I}_{\text {no drug, recover }}-\overbrace{j \alpha_{j} \rho I}^{\text {drug, deceased }}-\underbrace{j\left(1-\alpha_{j}\right) \gamma_{j} I}_{\text {drug, recover }}
$$

where $\alpha_{j}$ is the proportion of individuals who die despite receiving the drug. Likewise, $\gamma_{j}$ is the rate at which the population treated with the drug recovers. It is assumed that the time it takes to die does not depend on receiving the drug so $\rho$ does not change. With these new additions, the change in the recovered population becomes the following:

$$
\frac{d R}{d t}=(1-\mu) A n_{r}+(1-j)(1-\alpha) \gamma I+j\left(1-\alpha_{j}\right) \gamma_{j} I
$$

The change in the deceased population becomes the following:

$$
\frac{d D}{d t}=(1-j) \alpha \rho I+j \alpha_{j} \rho I
$$

Overall, the equations (2.1), (2.2) and (3.1)-(3.3) describe the population change with the introduction of a drug (Appendix B).

3.2. Tuning Parameters. Because antiviral drug trials are still underway, data about their effectiveness is not available. Both influenza and COVID-19 are respiratory viruses and share many symptoms and complications. Therefore, as a heuristic, we will begin by using data from influenza antivirals as baseline constants. We only use these data as baselines and run our model with different values through a sensitivity analysis.

The parameters $\beta_{a}, \beta_{i}, \mu, n_{i}, n_{r}, \alpha, \gamma$ and $\rho$ remain the same from the Basis Model. The proportion of individuals who receive the drug and die, $\alpha_{j}$, is estimated at half of $\alpha$ based on the effectivenesses of other antiviral drugs [12]. Therefore, $\alpha_{j}$ is $0.32 \%$.

Influenza antivirals can help sick people recover, on average, in 6.8 days [14]. This is a $14.7 \%$ reduction in the diseases' duration. We use this fraction along with the previous duration of 11.5 days to estimate $\gamma_{j}$ as $\frac{1}{11.5 \times(1-0.147)}=\frac{1}{9.810}=0.102$.

These parameters can be summarized in Appendix D.

3.3. Drug Availability. For the availability of the drug, we use a logistic curve to model the percentage of patients who are treated with the drug at time $t$ :

$$
j(t)=\frac{1}{1+e^{a(-t+b)}}
$$

Logistic growth is reasonable because manufacturing will ramp up exponentially at the beginning of production, while towards the end of its distribution, difficult access to rural communities or those with poor access to healthcare will slow growth.

In Figure $5, a=0.1$ is the logistic growth rate of the curve and $b=200$ (arbitrary) is the inflection point. For the purpose of this model, $a=0.1$ has been chosen so that the majority of the change in drug distribution occurs over a span of 30 days. Here $j(215)-j(185) \approx 0.635$. We feel this is a reasonable time span based on the COVID19 response. In practice, small changes in the value of $a$ do not change our final 


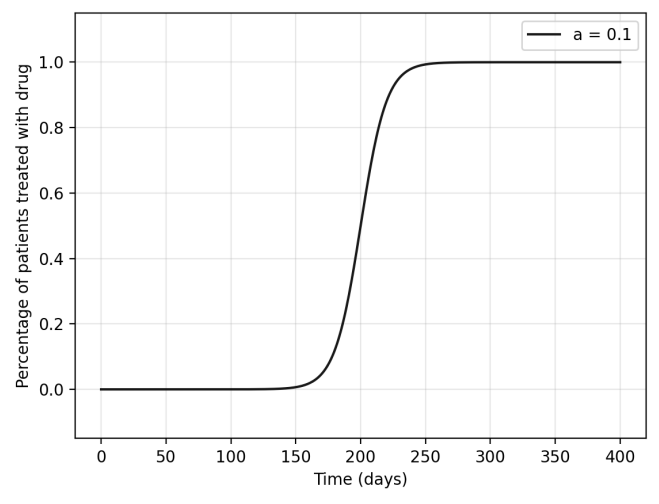

FIG. 5. Drug Distribution j(t)

analysis or conclusion. This is the case as long as the distribution takes place within the duration of the outbreak itself (i.e. $\sim 200$ days [see Figure 3] which corresponds to roughly $a \geq 0.04$ ). In the remainder of the paper when the term 'release date' of the drug or vaccine is used, we are referring to the inflection point of the curve.

3.4. Equilibrium. While equilibrium is typically related to autonomous systems, the time dependence of particular parameters is alleviated because it is assumed these variables become constants as time approaches infinity. As before, $n_{i}$, $n_{r}, \rho, \gamma, \gamma_{j}, \beta_{a}$ and $\beta_{i}$ are greater than zero. Also, $\mu, \alpha$, and $\alpha_{j}$ are greater than zero and less than one. Furthermore, $j(t)$ is a logistic curve bounded by 0 and 1 . Based on these constraints, the model follows the same structure as the Basis Model. Thus, $I^{*}=A^{*}=0$ at equilibrium. $S^{*}, R^{*}$, and $D^{*}$ can be any constants such that $S^{*}+R^{*}+D^{*}=N$. In summary, similar to the Basis Model, the equilibrium point $\boldsymbol{u}^{*}=\left[S^{*}, 0,0, R^{*}, D^{*}\right]^{T}$.

To understand the stability, $j(t)$ is set to its asymptotic value of 1 since it is assumed the drug would be fully distributed by the equilibrium where there are no symptomatically infected individuals. In solving for the eigenvalues, three are found to equal zero. One eigenvalue is negative. The final eigenvalue is positive when $\frac{S_{0}}{N}>0.48$. Therefore, the outbreak would have been prevented if $S_{0}$ represented less than $48 \%$ of the total population, given that the drug was already fully available $(j(t)=1)$. Overall, the drug does not change the nature of the equilibrium, merely the values.

3.5. Parameter Sensitivity Analysis. This section investigates how varying $\gamma_{j}$ (the rate at which people recover with drug) and $\alpha_{j}$ (the proportion of people who receive the drug and die) impacts the final death toll (i.e. $\lim _{t \rightarrow \infty} D(t)$ ). Figures 6 and 7 plot the release date of the drug against the final death toll. Each graph varies $\gamma_{j}$ and $\alpha_{j}$ while the other remains constant at $\alpha_{j}=\alpha$ or $\gamma_{j}=\gamma$. The horizontal line at the top of each figure remains constant because neither $\gamma$ nor $\alpha$ have changed, so the drug has no effect. In both figures, each curve converges to the same final death toll because the drug has no effect if it is released after the pandemic has passed. In both figures, the inflection point of each curve aligns with the peak of infections.

In Figure 6, as we expect, the earlier that the drug is released, the lower the death toll where each curve is roughly sigmoidal. For early release dates, the more effective (i.e. faster recovery time) drugs save many more lives than the less effective drugs. 


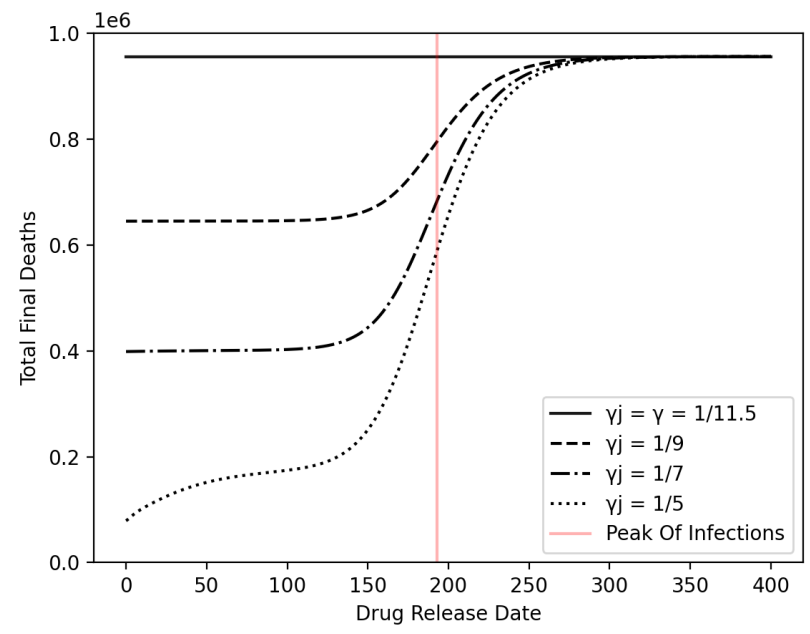

FIG. 6. Final Death Toll vs. Release Date, Varying $\gamma_{j}$

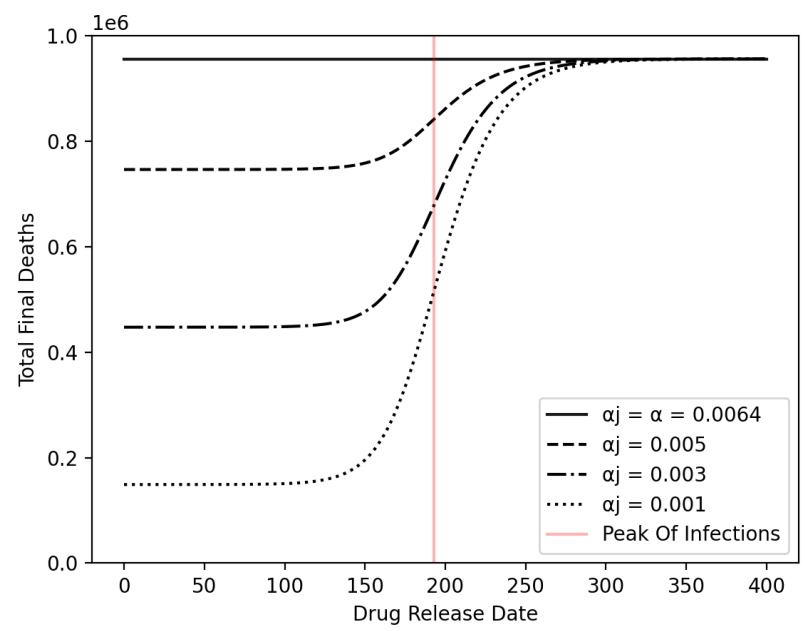

Fig. 7. Final Death Toll vs. Release Date, Varying $\alpha_{j}$

However, as the release date nears the peak of infections, this difference in outcome shrinks. Finally, it is not clear why the $\gamma_{j}=1 / 5$ curve "tapers off" as $t \rightarrow 0^{-}$.

In Figure 7 , if the drug is released on day 1 , any change in $\alpha_{j}$ is met with a proportional change in the final deaths (see $\alpha_{j}=0.005,0.003,0.001$ ). Again, we see sigmoidal curves where earlier release dates save more lives.

Our model suggests that while substantial benefit can still be derived from releasing a drug after the peak of infections, the death toll will be reduced dramatically if the drug is released far before the peak. However, a 'good' drug can save more lives if released after the peak than a comparatively 'bad' drug available from the beginning.

\section{Inclusion of a Vaccine.}

4.1. Modeling the Vaccine. Now we explore the inclusion of a vaccine on the system of equations. We will not simultaneously model the distribution of a drug and vaccine, but do compare the two in section 5. Therefore we will come back to 
our original Basis Model (section 2) as a starting point. There are a handful of ways to incorporate vaccinations into an SIR type model. One strategy alters the initial recovered population $\left(R_{0}\right)$ to represent those originally "vaccinated" [21]. Another vaccinates a fraction of newborns as they are introduced into the population [22]. Finally, one could subtract individuals from the susceptible population and move them into the recovered category. This negative rate of change could be in proportion to the size of the susceptible population or independent of it. For this model we remove $v(t)$ individuals from the susceptible population each day. We define $v(t)$ as the number of vaccinations distributed on a given day, independent of the size of the susceptible population. This approach is best for our modeling purposes because we can control exactly when, how quickly, and how many vaccinations are distributed. This would be less intuitive with a vaccination rate proportional to the susceptible population. The major assumption underlying this modeling decision is that successfully vaccinated individuals will become immediately and totally immune to the virus.

That being said, vaccinations are not always successful. If one was unsuccessful, the recipient would not know and would not become vaccinated again [16]. Therefore, the susceptible population is split into two different groups: The original susceptible population $(S)$, and the population of who received an ineffective vaccination $\left(S_{i v}\right)$. For the overall dynamics of the model, this means that in addition to susceptible individuals moving into the asymptotically infected category $(A)$, they could also move directly into $S_{i v}$ or $R$. Finally, members of the $S_{i v}$ population can only move to $A$, analogous to the susceptible population of section 2 . Figure 8 shows the dynamics of this new system and the constant total number of individuals $(N)$ becomes the following:

$$
N=S+S_{i v}+A+I+R+D
$$

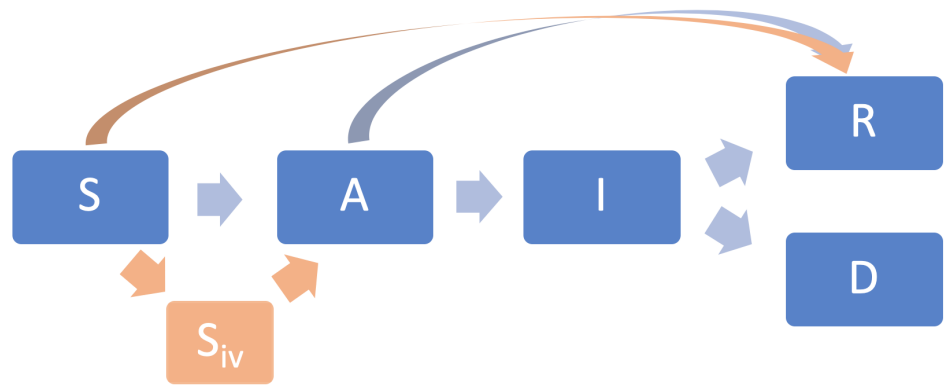

Fig. 8. Compartmental Flow Chart With Inclusion of Vaccine

One issue in removing $v(t)$ individuals each day surfaces as $S$ nears zero. A naive equation including $v(t)$ would look something like equation (2.1) minus $v(t)$ : $\frac{d S}{d t}=$ $-\left[\beta_{i} I\left(\frac{S}{N}\right)+\beta_{a} A\left(\frac{S}{N}\right)\right]-v$. Notice that because $v$ is a constant and not proportional to $S$ there is nothing stopping $S$ from becoming negative. Dealing with this boundary is important to ensure $N$ remains constant and people who never existed are not "vaccinated" and added to $S_{i v}$ or $R$. To address this issue, we alter our equations when $S$ nears zero using a Heaviside step function (4.1). In discrete terms, for the sake of example, it is clear $S$ is nearing zero when $\left|\frac{d S}{d t}\right|>S$ because this suggests during the next day $S$ will become negative (remember $\frac{d S}{d t}$ is strictly negative and $S$ 
is positive). Therefore we choose to split our equations at this point. ${ }^{1}$

Before we continue, note that $\left|\frac{d S}{d t}\right|=\beta_{i} I\left(\frac{S}{N}\right)+\beta_{a} A\left(\frac{S}{N}\right)+v>S$ can be rearranged to become $v>S-\left[\beta_{i} I\left(\frac{S}{N}\right)+\beta_{a} A\left(\frac{S}{N}\right)\right]$, i.e. when the number of vaccinations becomes larger than $S$ minus those transitioning into $A$. In the beginning, when $v$ is less than this value, we use the "naive" implementation described above. After this point we set $\frac{d S}{d t}=-S$, vaccinating the remaining population of $S$ with exponential decay, ensuring it never becomes negative. Given all of this, the Heaviside function becomes the following: ${ }^{2}$

$$
H= \begin{cases}1 & \text { if } v \leq S-\left[\beta_{i} I\left(\frac{S}{N}\right)+\beta_{a} A\left(\frac{S}{N}\right)\right] \\ 0 & \text { otherwise }\end{cases}
$$

With the inclusion of this function, $H$, the change in the population of susceptible individuals is modeled by the following:

$$
\frac{d S}{d t}=-\left[\beta_{i} I\left(\frac{S}{N}\right)+\beta_{a} A\left(\frac{S}{N}\right)\right] H-(v-S) H-S
$$

Notice that when $H=1$, the equation becomes $\frac{d S}{d t}=-\left[\beta_{i} I\left(\frac{S}{N}\right)+\beta_{a} A\left(\frac{S}{N}\right)\right]-v$ (equation (2.1) minus $v$ ). When $H=0$, the equation becomes $\frac{d S}{d t}=-S$.

As vaccinated individuals are removed from $S$, they move either to $S_{i v}$ or $A$ based on $\kappa$, the vaccine's effectiveness. The change in the new compartment, susceptible but ineffectively vaccinated is modeled by the following:

$$
\frac{d S_{i v}}{d t}=[(1-\kappa) v-(1-\kappa) S] H+(1-\kappa) S-\left[\beta_{i} I\left(\frac{S_{i v}}{N}\right)+\beta_{a} A\left(\frac{S_{i v}}{N}\right)\right]
$$

where $(1-\kappa)$ is the percentage of ineffective vaccinations. The two positive terms (new ineffective vaccinations) are $(1-\kappa) v$ and $(1-\kappa) S$ for $H=1$ and $H=0$, respectively. Regardless of $H$, the negative term, $-\left[\beta_{i} I\left(\frac{S_{i v}}{N}\right)+\beta_{a} A\left(\frac{S_{i v}}{N}\right)\right]$, remains the same. Again this is the same as equation (2.1).

The change in the asymptomatic population now includes a positive term for ineffectively vaccinated individuals who become infected. The first term disappears when $H=0$ because the entire susceptible population is vaccinated and no one new becomes infected.

$\frac{d A}{d t}=\left[\beta_{i} I\left(\frac{S}{N}\right)+\beta_{a} A\left(\frac{S}{N}\right)\right] H+\left[\beta_{i} I\left(\frac{S_{i v}}{N}\right)+\beta_{a} A\left(\frac{S_{i v}}{N}\right)\right]-\mu A n_{i}-(1-\mu) A n_{r}$

The symptomatically infected population equation, $\frac{d I}{d t}$, remains the same as that of the Basis Model equation (2.3). The change in the recovered population is very similar to equation (2.4) from the Basis Model, with the addition of those who receive

\footnotetext{
${ }^{1}$ Attempts were made to mark this break point at $S=0$ and then set $\frac{d S}{d t}=0$, but the resulting kink leads to issues with numerical integration.

${ }^{2}$ Despite the amount of thought put into the boundary at $S=0$, its overall impact on the model is next to none as it is only deals with the last tiny percentage of the susceptible population. Therefore, $S$ 's decay after the Heaviside function flips (e.g. linear, exponential or instantaneous) has no discernible effect on the outcome of the simulation. That being said, its implementation does matter when considering kinks or discontinuity and therefore issues with numerical integration. The choice of exponential decay avoids these issues.
} 
a successful vaccination. This will either be $\kappa v$ or $\kappa S$ depending on the Heaviside function:

$$
\frac{d R}{d t}=(1-\mu) A n_{r}+(1-\alpha) \gamma I+(\kappa v-\kappa S) H+\kappa S
$$

We assume that a vaccination does not affect the rate at which people die, so $\frac{d D}{d t}$ remains the same as that of the Basis Model equation (2.5). Overall, the equations (2.3), (2.5) and (4.2) - (4.5) (Appendix C) describe the population change with the introduction of a vaccine.

4.2. Tuning Parameters. For the sake of this model, we assume that the SARS-CoV-2 vaccine will consist of a single dose and will grant lasting immunity (i.e. the virus will not mutate significantly). Therefore, $\kappa$, the effectiveness of the vaccine is estimated by averaging the effectiveness of viral vaccines with lasting immunity. We estimate the vaccine to be $91 \%$ effective averaging eight known effectivenesses of common viral vaccines [16]. Again, $\beta_{a}, \beta_{i}, \mu, n_{i}, n_{r}, \alpha, \gamma$ and $\rho$ are all estimated using the values from the Basis Model. A summary of the value of these parameters is presented in Appendix D.

4.3. Vaccine Availability. For the release of the vaccine, a logistic distribution is used to model the number of new vaccinations available to be administered to susceptible individuals at time $t$ :

$$
v(t)=q \frac{a e^{a(-t+b)}}{\left(1+e^{a(-t+b)}\right)^{2}}
$$

Here, $q$ is the total number of vaccines distributed, $a$ is the logistic growth rate, and $b$ is the date of maximum vaccine distribution growth.

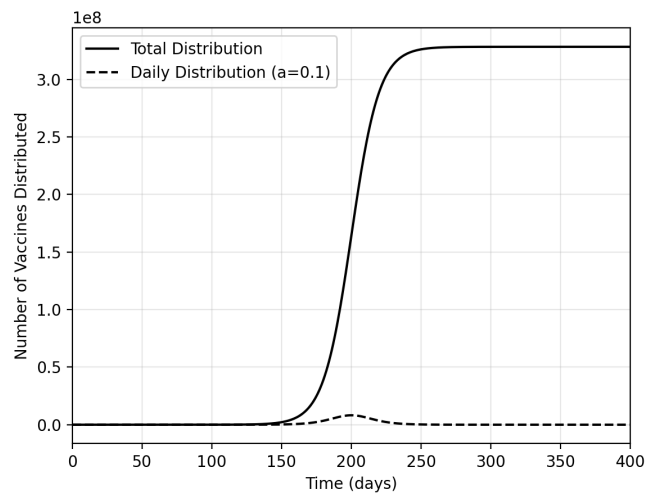

FIG. 9. Total/Daily Vaccine Distribution

Figure 9, shows the total (solid) and daily (dashed) number of vaccines distributed. The dashed curve, $v(t)$, is the derivative of the solid curve. Note that for the sake of this figure, the final number of vaccines distributed is set at the U.S. population size. However the following simulations are stratified for different percentages of total population vaccinated. Again, like the drug, $a=0.1$ has been chosen so that the majority of the vaccinations are distributed in a month-long span. As with the drug, from testing, the exact value of $a$ has little effect on our analysis. 
4.4. Equilibrium. As $t$ continues to infinity, $v(t)$ approaches zero. Therefore, the system loses its time-dependence. In this model, $n_{i}, n_{r}, \rho, \gamma, \beta_{a}$, and $\beta_{i}$ are greater than zero. Similar to before, $\mu, \alpha$, and $\kappa$ are also greater than zero and less than one. Given these constraints, $I^{*}$ must be zero so that $\frac{d D}{d t}$ is in equilibrium (equation (2.5)). Furthermore, $A^{*}$ must also be zero so that $\frac{d I}{d t}$ is in equilibrium (equation $(2.3)$ ). With $I^{*}$ and $A^{*}$ set to zero, $\frac{d A}{d t}=\frac{d I}{d t}=\frac{d D}{d t}=0$. To satisfy $\frac{d R}{d t}=0$ (equation (4.5)), $v(t)$ or $S^{*}$ must be zero, based on the Heaviside equation. If the Heaviside is always equal to 1 , then, as noted, $v(t)$ approaches zero as $t$ approaches infinity. We focus on the second case, when the Heaviside equals 0 and thus where $S^{*}$ is zero. After the Heaviside function flips, $S^{*}$ decays exponentially to zero. Therefore, an equilibrium point is achieved when $A^{*}$ and $I^{*}$ are zero and the Heaviside function has flipped, i.e. $v>S . S_{i v}^{*}, R^{*}$, and $D^{*}$ can be any constants such that $S_{i v}^{*}+R^{*}+D^{*}=N$. Overall, an equilibrium point, $\hat{\boldsymbol{u}}^{*}$, is achieved at $\left[0, S_{i v}^{*}, 0,0, R^{*}, D^{*}\right]^{T}$.

Based on the estimated values for the constants, the first four of the the eigenvalues are $-1,0,0$, and 0 . The fifth eigenvalue is negative. The sixth eigenvalue is positive when $\frac{S_{(i v) 0}}{N}>0.52$. Similar to the Basis and Drug models, when $S_{(i v) 0}$ represents less than $52 \%$ of the total population, the sixth eigenvalue is negative and the initial conditions are stable.

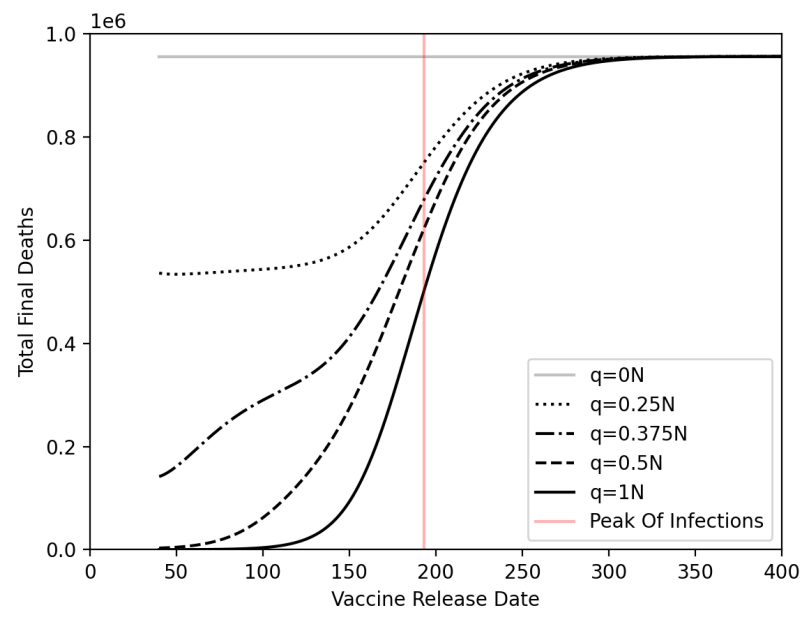

Fig. 10. Final Death Toll vs. Vaccine Release Date, Varying Population Vaccination Percentage

4.5. Parameter Sensitivity Analysis. Just as subsection 3.5 analyzed the effect of the drug's release date on death toll, this section analyzes that of the vaccine. Figure 10 is stratified for $q$, or the total number of vaccinations available for distribution, whereas Figure 11 is stratified for $\kappa$, or the perfect effectiveness of the vaccine. In Figure 10, $\kappa$ is held constant at our estimated value of 0.91 and in Figure $11, q$ is held constant at $N$ (i.e. the total population). Remember that we refer to the inflection point of the distribution of vaccines as their 'release date'. Therefore each curve begins slightly after day zero so that for the earliest release date all of the vaccinations are still distributed.

In Figure 10 each curve has a positive slope and is roughly sigmoidal. However, near time 0 , the curves tend to bend downward and to the left (see $\kappa=0.375$ ). This is likely because, for early release dates, a majority of vaccinations are administered before infections start to ramp up. Therefore the population can reach herd immunity 
before the virus has a chance to spread exponentially.

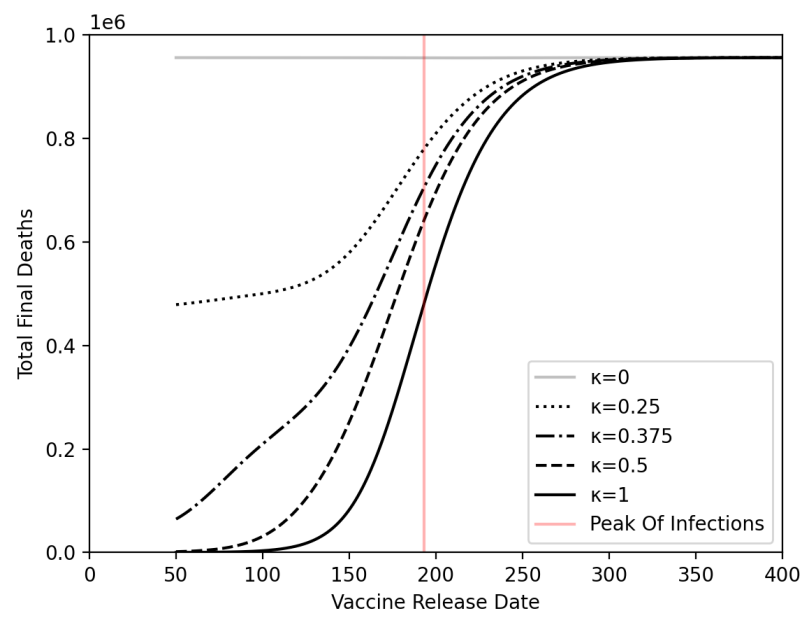

Fig. 11. Final Death Toll vs. Vaccine Release Date, Varying Vaccine Effectiveness

Figure 11 shows how vaccine effectiveness, $\kappa$, impacts the death toll. The figure is similar to Figure 10 because $q=N$ and vaccinating the entire population with a $50 \%$ effective vaccine is essentially the same as vaccinating $50 \%$ of the population with a $100 \%$ effective vaccine. However, in Figure 10, $\kappa=91 \%$ not $100 \%$ so the figures are similar but are not identical. For both figures, it is important to note how the difference in outcome shrinks as the release date is delayed. For instance, the difference in outcome between the $25 \%$ and $50 \%$ effective vaccinations shrinks from $5 e 5$ deaths from the beginning to less than $1 e 5$ at the peak of infections.

\section{Final Results.}

5.1. Antiviral Drugs and Vaccines. Based on the analysis in Section 4.5, the best time to release a drug or vaccine is far before the peak of the pandemic itself. At and after the peak of infections, the outcome quickly worsens. That being said, many times a relatively 'good' countermeasure saves more lives if released at the peak of infections compared to a 'bad' countermeasure released long before. This section directly compares the effects of a drug and vaccine.

Figure 12 compares a perfect drug and a perfect vaccine while Figure 13 compares a reasonable drug and reasonable vaccine (with added variation in total distribution). The perfect drug reduces the death rate to $0 \%$, the recovery time becomes instant, and every symptomatically infected patient receives it. The perfect vaccine is $100 \%$ effective and has $100 \%$ vaccination capacity (i.e. $\mathrm{N}$ vaccines are available for distribution). The reasonable drug and vaccine use the variables and values that were researched and discussed in previous sections. About $50 \%$ of the U.S. population receives the flu vaccine each year [1]. Therefore we have chosen $50 \%, 70 \%$, and $90 \%$ as capacity values. In both figures, $a$ or the speed of distribution of the two countermeasures is equal.

In Figure 12, the perfect drug has fewer total deaths compared to the perfect vaccine over the entire span of potential release dates. This makes sense intuitively. A vaccine can not save people who are already infected. If the vaccine were released during the peak of the pandemic, fewer people would benefit. However, the drug can help those already in the symptomatically infected category. Additionally, with every 
SIR MODEL OF TIME DEPENDENT DRUG/VACCINE DISTRIBUTION ON COVID-19

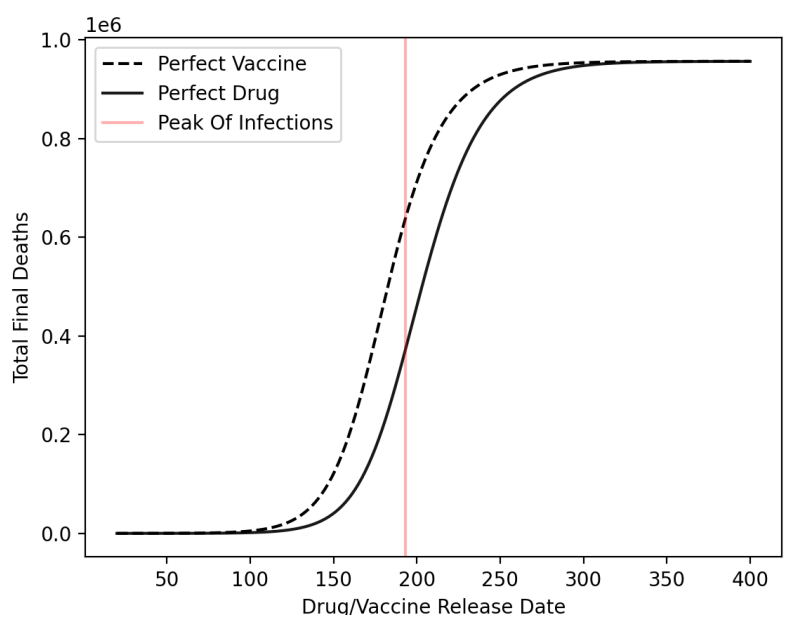

FIg. 12. Final Death Toll vs. Perfect Drug/Vaccine Release Date

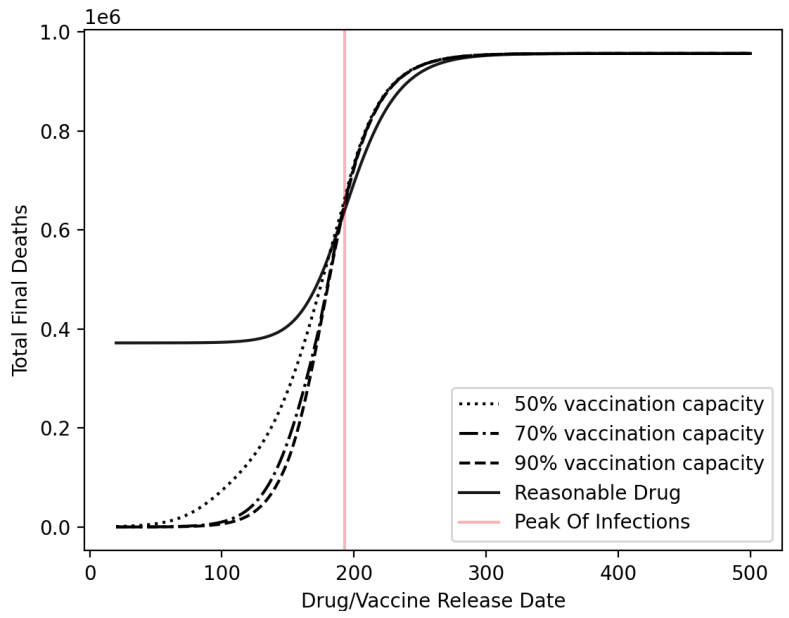

Fig. 13. Final Death Toll vs. Reasonable Drug/Vaccine Release Date

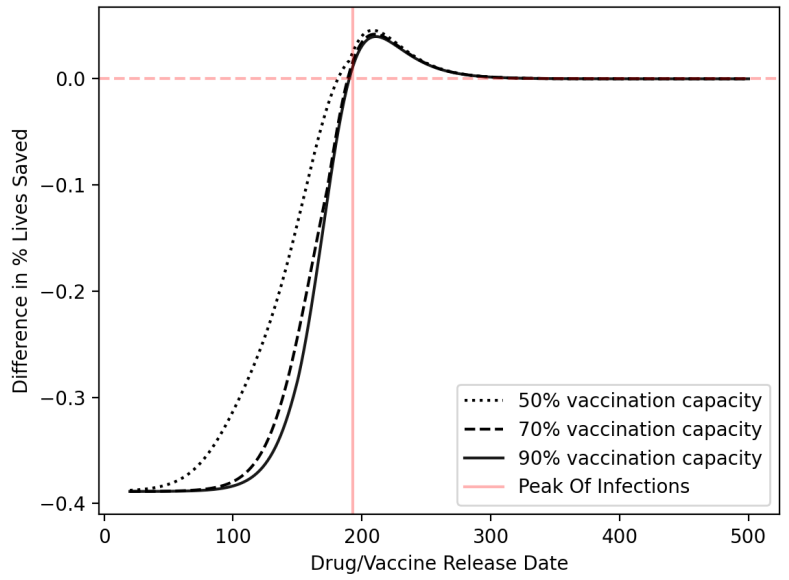

FIG. 14. Difference in \% of Lives Saved (Drug - Vaccines) 
symptomatically infected person receiving it and immediately recovering, the ability for the virus to spread is severely diminished.

In Figure 13, every reasonable vaccine vastly outperforms the reasonable drug when released before the peak of infections, while the reasonable drug slightly outperforms each vaccine following the peak of infections. This follows similar logic to the first graph. The vaccine is much more effective when released early because it grants the population herd immunity, but it is not as helpful after many are infected, whereas a drug is.

Figure 14 presents the same information as Figure 13, but directly compares the percentage of lives saved by the drug and the vaccine. To construct this graph, first we find how effective each countermeasure is in terms of percentage of total deaths reduced. Total deaths without any intervention is 956,222. To calculate percentage of total deaths reduced for each countermeasure we use the equation 1 - (deaths/956,222), where deaths is the total final deaths for that countermeasure if it is released at the given time. Finally, we subtract the percentage of lives saved by each vaccine from that of the drug to give us the y-axis. Interpreting this graph, a $y$-axis value of 0 means that the countermeasures are equally as effective, whereas a positive value of 0.1 means that the drug saved $10 \%$ more lives than the vaccine.

Analysis of Figure 14 finds that at time zero (i.e. the drug and vaccine are available since the beginning), each vaccine saves $\sim 39 \%$ more lives than the drug. However shortly after the peak of infections, each curve reaches a maximum at $0.0457 \%$, $0.0421 \%$, and $0.0398 \%$ for the $50 \%, 70 \%$, and $90 \%$ distribution brackets respectively. This means that at its best, the drug saves between $4 \%$ and $4.6 \%$ more lives when released at that time. If each countermeasure is released directly at the peak of infections, the drug only performs slightly better than the vaccine, saving $2.53 \%, 1.64 \%$, and $1.17 \%$, more lives than the vaccine at the $50 \%, 70 \%$, and $90 \%$ distribution brackets.

\section{Discussion.}

6.1. Equilibrium Analysis. The introduction of a drug does not change the equilibrium point, as both the Basis and the Drug Models are in equilibrium when $A^{*}$ and $I^{*}$ are zero. However, with the introduction of a vaccine, equilibrium is only reached when $S^{*}$ as well as $A^{*}$ and $I^{*}$ are zero. In general, all of the equilibrium points are asymptotically stable when $S$ or $S_{i v}$ is small enough. For the Basis and Vaccine Models, $S_{0}$ and $S_{(i v) 0}$ must be less than $52 \%$ of the population, respectively. This indicates herd immunity would have been achieved if, at the outbreak, about half of the population was already immune. If the drug had been available since the beginning of the outbreak, this value would be slightly lower at $48 \%$. Overall, the three models are similar, and the introduction of a drug or vaccine does not significantly change the equilibrium and its stability.

6.2. Limitations. Our model's first and most significant limitation is its basis as an SIR model. While we would expect, and do observe, 'real-world' infection totals to grow and ultimately saturate, the logistic distribution is never a perfect fit. There are many more factors at play in a pandemic that cannot be fully explained by a simple system of equations. Unpredictable, exogenous factors include the availability of medical supplies, hospital capacity, social distancing, and municipal lock-downs, among others. SIR models also treat every person the same, neglecting risk factors (e.g. age, obesity), geography (urban vs. rural), and gender, among other personal 
characteristics.

Further, our recovered population cannot be re-infected. Research shows that if individuals recover from SARS-CoV-2, they are at risk for re-infection after about 160 days [10], though the risk is lower. As more data becomes available, it may be necessary to return members of the recovered population to the susceptible population after a certain amount of time. In doing this, it would be necessary to have separate populations of 'recovered via the vaccine' and 'recovered after infection' because the re-infection rate would be lower for vaccinated populations.

Another limitation arises in fixing the total population $(N)$. Our model ignores natural deaths due to causes other than COVID-19 and new people's entrance, such as births or immigration. In the long run, people who gained immunity would be replaced by newborns, who are susceptible to the virus. However, since this paper mostly analyzed the effect of drugs and vaccines, we assumed that the period would be short enough to ignore these long-term factors.

Other limitations arise from our heuristics. In our Basis Model this includes using a constant $R_{0}$, using uniform disease duration to calculate $\beta$, and assuming asymptomatic people are equally contagious to those who are symptomatic $\left(\beta_{i}=\right.$ $\beta_{a}$ ). With the inclusion of the vaccine, we assume that (if effective) a single dose immediately places an individual in the recovered population.

For all of these reasons, the final number of the total deaths should not be extrapolated. However, general trends and relationships between drugs and vaccine distribution are captured by our model.

6.3. Possible Next Steps. First potential next steps would include fixing and adding elements from the limitations section. It may be possible to forecast and use historical $R_{t}$ data to make $\beta_{a}$ and $\beta_{i}$ time dependent variables. This would require more data about differences in infectivity between asymptomatic and symptomatically infected populations as well as some idea about future social distancing and its impacts on $R_{t}$. Further, subdividing the symptomatically infected and asymptomatic populations into at risk groups would also bring the model closer to reality. These risk groups could include the elderly, the immunocompromised, and the obese, among others [5].

Another possible next step would have to do with increasing case counts in the U.S. and a potential second wave. The projected release dates for many vaccines and Operation Warp Speed come around January 2021. This could coincide with a second wave after people return to school and work in the fall. While the details would be much more complex, a second wave is on many Americans' minds right now and is as important as ever.

It would also be interesting to test how varying the speed of each countermeasure's distribution affects our analysis. In section 5 , we assume the speed of distribution for the two is equal. However it could be the case that a vaccination's distribution could take longer than that of a simple pill.

Finally, as new vaccines and drugs are developed, more data about their specific effectivenesses will be available. It would be interesting to plug those values directly into the model to compare each vaccine and drug along with each of their projected release dates to observe different outcomes.

6.4. Conclusion and Perspectives. From analysis of our model, the best time to release a drug or vaccine is long before a pandemic begins. Each day that a countermeasure's release is delayed many more lives are lost. For the early distribution dates of a drug, changes in effectiveness result in proportional changes in the final 
death toll. However for early release dates of a vaccine, this is not the case. The rate of return on the number of vaccines distributed increases, consistent with what we know about herd immunity.

In comparing the release of a drug and vaccine directly, we find that long before the peak of infections, vaccines are more effective. At and after the peak of infections, the release of a drug will save marginally more lives. If a vaccine or drug were available for the entire duration of the disease, about $40 \%$ more lives would be saved with a vaccine. However, after the peak of infections, a drug can save, at most, $\sim 4 \%-4.6 \%$ more lives depending on how many vaccines are distributed.

Our results are not to suggest the prioritization of one countermeasure over another in the U.S's response to the COVID-19 pandemic. However, they do suggest how critical it is to slow down the virus and buy time for the development of countermeasures.

\section{REFERENCES}

[1] Seasonal Influenza Vaccine Supply for the U.S. 2019-2020 Influenza Season, 2019, https://www.cdc.gov/flu/prevent/vaxsupply.htm.

[2] Fact Sheet: Explaining Operation Warp Speed, 2020, https://www.hhs.gov/about/news/ 2020/06/16/fact-sheet-explaining-operation-warp-speed.html.

[3] Interim Clinical Guidance for Management of Patients with Confirmed Coronavirus Disease (COVID-19), 2020, https://www.cdc.gov/coronavirus/2019-ncov/hcp/ clinical-guidance-management-patients.html.

[4] Novel coronavirus (covid-19) cases data, July 2020, https://data.humdata.org/dataset/novel-coronavirus-2019-ncov-cases.

[5] People with Certain Medical Conditions, 2020, https://www.cdc.gov/coronavirus/2019-ncov/ need-extra-precautions/people-with-medical-conditions.html.

[6] Report of the WHO-China Joint Mission on Coronavirus Disease 2019 (Covid-19). https://www.who.int/docs/default-source/coronaviruse/ who-china-joint-mission-on-covid-19-final-report.pdf, 2020.

[7] D. Armstrong and E. Pfanner, Coronavirus Vaccine Tracker: Race for a Covid-19 Cure. https://www.bloomberg.com/features/2020-coronavirus-drug-vaccine-status/, 2020.

[8] D. C. Buitrago-Garcia, D. Egli-Gany, M. J. Counotte, S. Hossmann, H. Imeri, A. M. IPEKCI, G. SAlanti, AND N. Low, The role of asymptomatic SARS-CoV-2 infections: rapid living systematic review and meta-analysis, medRxiv, (2020), https://doi.org/10.1101/2020.04.25.20079103, https://www.medrxiv.org/content/early/2020/05/24/2020.04.25.20079103.

[9] O. Byambasuren, M. Cardona, K. Bell, J. Clark, M.-L. Mclaws, and P. Glasziou, Estimating the extent of asymptomatic COVID-19 and its potential for community transmission: systematic review and meta-analysis, medRxiv, (2020), https://doi.org/10.1101/2020.05.10.20097543, https://www.medrxiv.org/content/early/2020/06/04/2020.05.10.20097543.

[10] N. Davis, What we know about Covid reinfection, immunity and vaccines, 2021, https://www.theguardian.com/world/2021/feb/12/ what-we-know-about-covid-reinfection-immunity-and-vaccines.

[11] H. Froese, Infectious Disease Modelling: Beyond the Basic SIR Model. https://www.towardsdatascience.com/ infectious-disease-modelling-beyond-the-basic-sir-model-216369c584c4, 2020

[12] R. Gani, H. Hughes, D. Fleming, T. Griffin, J. Medlock, and S. Leach, Potential impact of antiviral drug use during influenza pandemic, Emerging infectious diseases, 11 (2005), p. 1355.

[13] S. A. Lauer, K. H. Grantz, Q. Bi, F. K. Jones, Q. Zheng, H. R. Meredith, A. S. Azman, N. G. REICH, AND J. Lessler, The incubation period of coronavirus disease 2019 (COVID-19) from publicly reported confirmed cases: estimation and application, Annals of internal medicine, 172 (2020), pp. 577-582.

[14] R. Lehnert, M. Pletz, A. Reuss, And T. Schaberg, Antiviral Medications in Seasonal and Pandemic Influenza: A Systematic Review, Deutsches Ärzteblatt International, 113 (2016), p. 799 . 
[15] G. Meyerowitz-Katz and L. Merone, A systematic review and meta-analysis of published research data on covid-19 infection-fatality rates, medRxiv, (2020), https://doi.org/10.1101/2020.05.03.20089854, https://www.medrxiv.org/content/early/2020/07/07/2020.05.03.20089854.

[16] R. REISs, Childhood vaccine effectiveness easy to use table, 2016, https://www.skepticalraptor.com/skepticalraptorblog.php/ childhood-vaccine-effectiveness-easy-use-table/.

[17] A. Sakurai, T. Sasaki, S. Kato, M. Hayashi, S.-I. Tsuzuki, T. Ishihara, M. Iwata, Z. Morise, And Y. DoI, Natural History of Asymptomatic SARS-CoV-2 Infection, New England Journal of Medicine, (2020), https://doi.org/10.1056/NEJMc2013020, https://arxiv.org/abs/https://doi.org/10.1056/NEJMc2013020.

[18] D. Smith And L. Moore, The SIR Model for Spread of Disease - The Differential Equation Model, 2020, https://www.maa.org/press/periodicals/loci/joma/ the-sir-model-for-spread-of-disease-the-differential-equation-model.

[19] M. Smith, K. Yourish, S. Almukhtar, K. Collins, D. Ivory, And A. Harmon, Coronavirus (Covid-19) Data in the United States, 2020, https://github.com/nytimes/covid-19-data.

[20] R. Verity, L. C. Okell, I. Dorigatti, P. Winskill, C. Whittaker, N. Imai, G. Cuomo-Dannenburg, H. Thompson, P. G. Walker, H. Fu, et Al., Estimates of the severity of coronavirus disease 2019: a model-based analysis, The Lancet infectious diseases, (2020), https://doi.org/10.1016/S1473-3099(20)30243-7, https://www.thelancet.com/journals/laninf/article/PIIS1473-3099(20)30243-7.

[21] H. WEIss, The sir model and the foundations of public health, MATerials MATemátics, (2013), https://mat.uab.cat/matmat_antiga/PDFv2013/v2013n03.pdf.

[22] P. Yongzhen, L. Shuping, L. Changguo, And S. Chen, The effect of constant and pulse vaccination on an sir epidemic model with infectious period, Applied Mathematical Modelling, 35 (2011), pp. 3866-3878, https://doi.org/https://doi.org/10.1016/j.apm.2011.02.012, https://www.sciencedirect.com/science/article/pii/S0307904X11000692.

\section{Appendices}

\section{Appendix A. Basis Model: System of Differential Equations.}

$$
\frac{d S}{d t}=-\beta_{i} I\left(\frac{S}{N}\right)-\beta_{a} A\left(\frac{S}{N}\right)
$$

$$
\frac{d A}{d t}=\beta_{i} I\left(\frac{S}{N}\right)+\beta_{a} A\left(\frac{S}{N}\right)-\mu A n_{i}-(1-\mu) A n_{r}
$$

$$
\frac{d I}{d t}=\mu A n_{i}-\alpha \rho I-(1-\alpha) \gamma I
$$

$$
\frac{d R}{d t}=(1-\mu) A n_{r}+(1-\alpha) \gamma I
$$

$$
\frac{d D}{d t}=\alpha \rho I
$$




$$
\frac{d S}{d t}=-\beta_{i} I\left(\frac{S}{N}\right)-\beta_{a} A\left(\frac{S}{N}\right)
$$

$$
\frac{d A}{d t}=\beta_{i} I\left(\frac{S}{N}\right)+\beta_{a} A\left(\frac{S}{N}\right)-\mu A n_{i}-(1-\mu) A n_{r}
$$

$$
\frac{d I}{d t}=\mu A n_{i}-(1-j) \alpha \rho I-(1-j)(1-\alpha) \gamma I-j \alpha_{j} \rho I-j\left(1-\alpha_{j}\right) \gamma_{j} I
$$

$$
\frac{d R}{d t}=(1-\mu) A n_{r}+(1-j)(1-\alpha) \gamma I+j\left(1-\alpha_{j}\right) \gamma_{j} I
$$

$$
\frac{d D}{d t}=(1-j) \alpha \rho I+j \alpha_{j} \rho I
$$

Appendix C. Vaccine Model: System of Differential Equations.

$$
H= \begin{cases}1 & \text { if } v<S-\left[\beta_{i} I\left(\frac{S}{N}\right)+\beta_{a} A\left(\frac{S}{N}\right)\right] \\ 0 & \text { otherwise }\end{cases}
$$

$$
\frac{d S}{d t}=-\left[\beta_{i} I\left(\frac{S}{N}\right)+\beta_{a} A\left(\frac{S}{N}\right)\right] H-(v-S) H-S
$$

(C.3) $\frac{d S_{i v}}{d t}=[(1-\kappa) v-(1-\kappa) S] H+(1-\kappa) S-\left[\beta_{i} I\left(\frac{S_{i v}}{N}\right)+\beta_{a} A\left(\frac{S_{i v}}{N}\right)\right]$

(C.4)

$638 \frac{d A}{d t}=\left[\beta_{i} I\left(\frac{S}{N}\right)+\beta_{a} A\left(\frac{S}{N}\right)\right] H+\left[\beta_{i} I\left(\frac{S_{i v}}{N}\right)+\beta_{a} A\left(\frac{S_{i v}}{N}\right)\right]-\mu A n_{i}-(1-\mu) A n_{r}$

$$
\frac{d I}{d t}=\mu A n_{i}-\alpha \rho I-(1-\alpha) \gamma I
$$

$$
\frac{d R}{d t}=(1-\mu) A n_{r}+(1-\alpha) \gamma I+[\kappa v-\kappa S] H+\kappa S
$$

$$
\frac{d D}{d t}=\alpha \rho I
$$


TABLE 1

Parameter Summary

\begin{tabular}{|c|c|}
\hline$\beta_{i}=0.1205$ & $\begin{array}{c}\text { Expected number of people a symptomatically } \\
\text { infected person infects per day. }\end{array}$ \\
$\beta_{a}=0.1205$ & $\begin{array}{c}\text { Expected number of people an asymptomatic person } \\
\text { infects per day. }\end{array}$ \\
$\mathrm{n}_{i}=0.1961$ & Rate at which people transition from asymptomatic to \\
& symptomatically infected. \\
$\mathrm{n}_{r}=0.1111$ & Rate at which people transition from asymptomatic to recovered. \\
$\mu=0.85$ & Proportion of asymptomatic individuals who become \\
& symptomatically infected. \\
$\alpha=0.0064$ & Proportion of infected individuals who die. \\
$\gamma=0.0870$ & Rate at which infected people recover. \\
$\rho=0.0562$ & Rate at which infected people die. \\
$\alpha_{j}=0.0032$ & Proportion of individuals who receive the drug and die. \\
$\gamma_{j}=0.102$ & Rate at which people recover with the drug. \\
$\kappa=0.91$ & Proportion of effective vaccinations
\end{tabular}

Appendix E. Jacobian Matrix.

$$
J=\left[\begin{array}{lllll}
\frac{\partial S^{\prime}(t)}{\partial S} & \frac{\partial A^{\prime}(t)}{\partial S} & \frac{\partial I^{\prime}(t)}{\partial S} & \frac{\partial R^{\prime}(t)}{\partial S} & \frac{\partial D^{\prime}(t)}{\partial S} \\
\frac{\partial S^{\prime}(t)}{\partial A} & \frac{\partial A^{\prime}(t)}{\partial A} & \frac{\partial I^{\prime}(t)}{\partial A} & \frac{\partial R^{\prime}(t)}{\partial A} & \frac{\partial D^{\prime}(t)}{\partial A} \\
\frac{\partial S^{\prime}(t)}{\partial I} & \frac{\partial A^{\prime}(t)}{\partial I} & \frac{\partial I^{\prime}(t)}{\partial I} & \frac{\partial R^{\prime}(t)}{\partial I} & \frac{\partial D^{\prime}(t)}{\partial I} \\
\frac{\partial S^{\prime}(t)}{\partial R} & \frac{\partial A^{\prime}(t)}{\partial R} & \frac{\partial I^{\prime}(t)}{\partial R} & \frac{\partial R^{\prime}(t)}{\partial R} & \frac{\partial D^{\prime}(t)}{\partial R} \\
\frac{\partial S^{\prime}(t)}{\partial D} & \frac{\partial A^{\prime}(t)}{\partial D} & \frac{\partial I^{\prime}(t)}{\partial D} & \frac{\partial R^{2}(t)}{\partial D} & \frac{\partial D^{\prime}(t)}{\partial D}
\end{array}\right]
$$

$$
645 \quad(J-\lambda I)=\left[\begin{array}{ccccc}
-\lambda & -\frac{\beta_{a} S}{N} & -\frac{\beta_{i} S}{N} & 0 & 0 \\
0 & \frac{\beta_{a} S}{N}-\mu n_{i}-(1-\mu) n_{r}-\lambda & \frac{\beta_{i} S}{N} & 0 & 0 \\
0 & \mu n_{i} & -\alpha \rho-(1-\alpha) \gamma-\lambda & 0 & 0 \\
0 & (1-\mu) n_{r} & (1-\alpha) \gamma & -\lambda & 0 \\
0 & 0 & \alpha \rho & 0 & -\lambda
\end{array}\right]
$$

